\title{
Mechanism of E-cadherin lysosomal degradation
}

\section{Wen Jin Wu and Dianne S. Hirsch}

We read with great interest the Review by Yaron Mosesson, Gordon B. Mills and Yosef Yarden ${ }^{1}$ (Derailed endocytosis: an emerging feature of cancer. Nature Rev. Cancer 8, 835850 (2008)) as it is highly relevant to our research. Experimental evidence indicates that epithelial (E)-cadherin ubiquitylation and degradation is an important mechanism contributing to the metastasis of epitheliumderived cancers. We would like to bring to your attention three articles that provide experimental evidence in support of the model of E-cadherin lysosomal degradation that is proposed in FIG. 3 of this Review.

In the legend to FIG. 3, the authors state that, following growth factor-induced activation of SRC, "SRC tags E-cadherin with phosphotyrosine. This is followed by ubiquitylation of E-cadherin by an E3 ligase called Hakai, thereby biasing degradation of E-cadherin and diminishing recycling through RAB11-containing recycling endosomes." Within the text, the authors properly cited Fujita et al. ${ }^{2}$, who originally identified Hakai as an E3 ligase that ubiquitylates E-cadherin, leading to E-cadherin endocytosis. However, key studies that support the authors' proposed endocytic mechanisms underlying lysosomal degradation of E-cadherin leading to disruption of adherens junctions and loss of tumour cell polarity were not cited. These recent publications have significantly contributed to defining the mechanisms of lysosomal degradation of internalized E-cadherin. Palacios et al. ${ }^{3}$ provided evidence that, following SRC expression, E-cadherin is internalized and then shuttled to the lysosomes for degradation resulting in reduced recycling of E-cadherin back to the lateral membrane. This study also showed that E-cadherin ubiquitylation is essential for the shuttling of E-cadherin to lysosomes. The studies by Janda et al. ${ }^{4}$ indicate that early stages of epithelial-mesenchymal transition involve cooperative, post-translational downregulation of E-cadherin. Recently, Shen et al. ${ }^{5}$ reported that CDC42, a Ras family small GTP-binding protein, regulates E-cadherin ubiquitylation and lysosomal degradation through a pathway mediated by epidermal growth factor receptor followed by SRC. Data from this study support a model that activation of CDC42 contributes to the development of a mesenchyme-like phenotype in the well-differentiated breast cancer cells by targeting E-cadherin for lysosomal degradation. Taken together, these studies established the machinery of lysosomal degradation of internalized E-cadherin in response to mitogens and oncogenic signals and should be cited to provide experimental evidence to support the model proposed by Mosesson et al. in their Review.

Wen Jin Wu and Dianne S. Hirsch are at the Division of Monoclonal Antibodies, Office of Biotechnology Products, Office of Pharmaceutical Science, Center for Drug Evaluation and Research, United States Food and Drug Administration, Bethesda, Maryland 20892, USA. Correspondence to W.J.W. e-mail:wen.wu@fda.hhs.gov

Published online 16 January 2009

1. Mosesson, Y., Mills, G. B. \& Yarden, Y. Derailed endocytosis: an emerging feature of cancer. Nature Rev. Cancer 8, 835-850 (2008).

2. Fujita, Y. et al. Hakai, a c-Cbl-like protein, ubiquitinates and induces endocytosis of the E-cadherin complex. Nature Cell Biol. 4, 222-231 (2002)

3. Janda, E. et al. Raf plus TGF $\beta$-dependent EMT is initiated by endocytosis and lysosomal degradation of E-cadherin. Oncogene 25, 7117-7130 (2006).

4. Palacios, F., Tushir, J. S., Fujita, Y. and D'SouzaSchorey, C. Lysosomal targeting of E-cadherin: a unique mechanism for the down-regulation of cell-cell adhesion during epithelial to mesenchymal transitions. Mol. Cell. Biol. 25, 389-402 (2005).

5. Shen, Y., Hirsch, D. S., Sasiela, C. A. \& Wu, W. J. $\mathrm{Cdc} 42$ regulates $\mathrm{E}$-cadherin ubiquitination and degradation through an epidermal growth factor receptor to Src-mediated pathway. J. Biol. Chem. 283, 5127-5137 (2008). 